\title{
High-dose thiamine therapy for patients with type 2 diabetes and microalbuminuria: a randomised, double-blind placebo-controlled pilot study
}

\author{
N. Rabbani • S. S. Alam • S. Riaz • J. R. Larkin • \\ M. W. Akhtar • T. Shafi • P. J. Thornalley
}

Received: 22 September 2008 / Accepted: 22 October 2008 / Published online: 5 December 2008

(C) Springer-Verlag 2008

\begin{abstract}
Aims/hypothesis High-dose supplements of thiamine prevent the development of microalbuminuria in experimental diabetes. The aim of this pilot study was to assess whether oral supplements of thiamine could reverse microalbuminuria in patients with type 2 diabetes.

Methods Type 2 diabetic patients (21 male, 19 female) with microalbuminuria were recruited at the Diabetes Clinic, Sheikh Zayed Hospital, Lahore, Pakistan, and randomised to placebo and treatment arms. Randomisation was by central office in sequentially numbered opaque, sealed envelopes. Participants, caregivers and those assessing the outcomes were blinded to
\end{abstract}

N. Rabbani $\cdot$ J. R. Larkin $\cdot$ P. J. Thornalley $(\bowtie)$

Clinical Sciences Research Institute, Warwick Medical School,

University of Warwick, University Hospital,

Clifford Bridge Road,

Coventry CV2 2DX, UK

e-mail: P.J.Thornalley@warwick.ac.uk

\section{S. S. Alam}

Department of Pharmacology, Sheikh Zayed Federal Postgraduate

Medical Institute, Sheikh Zayed Hospital,

Lahore, Pakistan

\section{S. Riaz}

Department of Microbiology and Molecular Genetics,

University of the Punjab,

Lahore, Pakistan

M. W. Akhtar

School of Biological Sciences, University of the Punjab,

Lahore, Pakistan

T. Shafi

Department of Nephrology, Division of Medicine,

Sheikh Zayed Federal Postgraduate Medical Institute,

Lahore, Pakistan group assignment. Patients were given $3 \times 100 \mathrm{mg}$ capsules of thiamine or placebo per day for 3 months with a 2 month follow-up washout period. The primary endpoint was change in urinary albumin excretion (UAE). Other markers of renal and vascular dysfunction and plasma concentrations of thiamine were determined.

Results UAE was decreased in patients receiving thiamine therapy for 3 months with respect to baseline (median $-17.7 \mathrm{mg} / 24 \mathrm{~h} ; p<0.001, n=20)$. There was no significant decrease in UAE in patients receiving placebo after 3 months of therapy $(n=20)$. UAE was significantly lower in patients who had received thiamine therapy compared with those who had received placebo ( $30.1 \mathrm{vs} 35.5 \mathrm{mg} / 24 \mathrm{~h}, p<0.01$ ) but not at baseline. UAE continued to decrease in the 2 month washout period in both groups, but not significantly. There was no effect of thiamine treatment on glycaemic control, dyslipidaemia or BP. There were no adverse effects of therapy. Conclusions/interpretation In this pilot study, high-dose thiamine therapy produced a regression of UAE in type 2 diabetic patients with microalbuminuria. Thiamine supplements at high dose may provide improved therapy for early-stage diabetic nephropathy.

Trial registration: CTRI (India) CTRI/2008/091/000112

Funding: Pakistan Higher Education Commission

Keywords Diabetic complications · Diabetic nephropathy · Microalbuminuria $\cdot$ Thiamine Type 2 diabetes

\begin{tabular}{|c|c|}
\hline Abbreviati & \\
\hline sVCAM-1 & soluble vascular cell adhesion molecule- 1 \\
\hline UAE & urinary albumin excretion \\
\hline
\end{tabular}

Abbreviations

UAE urinary albumin excretion

von Willebrand factor 


\section{Introduction}

Diabetic nephropathy develops progressively over 5-40 years of diabetes [1]. A novel strategy to counter biochemical dysfunction linked to the development of diabetic nephropathy is high-dose thiamine therapy [2]. High-dose thiamine therapy prevented the development of diabetic nephropathy in experimental diabetes without improvement of glycaemic control [3]. Experimental diabetes was associated with thiamine deficiency characterised by a marked decrease in plasma thiamine concentration and decreased activity and expression of the thiamine-dependent enzyme transketolase in renal glomeruli [4]. Type 1 and type 2 diabetic patients showed similarly decreased plasma thiamine concentrations [5]. In this pilot study, we evaluated the effect of oral highdose supplements of thiamine on urinary albumin excretion (UAE), a marker of early-stage diabetic nephropathy, in type 2 diabetic patients with microalbuminuria.

\section{Methods}

Diabetic patients were recruited from patients attending the Diabetes Clinic at the Sheikh Zayed Hospital, Lahore, Pakistan between October and December 2006. The main inclusion criteria were: age 35-65 years, type 2 diabetes and persistent microalbuminuria (AER 30-299 mg/24 h), diabetes duration $\geq 5$ years, $\mathrm{HbA}_{1 \mathrm{c}} \leq 12.5 \%$, and BMI $19-40 \mathrm{~kg} / \mathrm{m}^{2}$. The main exclusion criteria were: significant comorbidities, known allergy or intolerance to thiamine, use of thiamine supplements, participation in an interventional study within 30 days, recipients of renal and/or pancreatic transplant and women who were pregnant or breast feeding or of child bearing potential not using adequate contraceptive precautions.

Forty patients were enrolled and randomly assigned (random number table method) to receive placebo or $3 \times 100 \mathrm{mg}$ tablets/day for 3 months followed by a 2 month washout period. Adverse effects of thiamine treatment were assessed by kidney function (serum creatinine and urea nitrogen) and liver function (serum alanine transaminase, aspartate transaminase and alkaline phosphatase activities and bilirubin) tests. In the placebo group, there were 11 male and nine female patients of $52.4 \pm 8.7$ years and BMI $28.3 \pm$ $4.4 \mathrm{~kg} / \mathrm{m}^{2}$. In the thiamine treatment group, there were ten male and ten female patients $(52.7 \pm 8.4$ years old and BMI $28.1 \pm 4.6 \mathrm{~kg} / \mathrm{m}^{2}$ ). At baseline and throughout the study, nine patients in the thiamine treatment group and three in the placebo group were receiving insulin therapy $(p<0.05)$. There were no other significant differences in the proportions of patients receiving therapeutic agents in the thiamine treatment and placebo groups. One patient achieved glycaemic control by diet only; all others received therapy with hypoglycaemic agents (sulfonylureas, metformin and thiazolidinediones).
Nine patients in the placebo group and eight in the thiamine treatment group received therapy with antihypertensive agents. Three patients in the thiamine treatment arm and one in the placebo arm were receiving cholesterol-lowering statin therapy, compared with seven and two respectively after 3 months, and seven and three after the 2 month washout period. One patient in the thiamine treatment group was receiving antihyperlipidaemic fibrate therapy.

Twenty-four hour urine collections and fasting blood samples were taken with the informed consent of the participants. Plasma and erythrocyte fractions were separated immediately and stored at $-80^{\circ} \mathrm{C}$ until analysis.

Ethical approval for the study was given by the Ethical/ Protocol/Synopsis Committee of Sheikh Zayed Federal Postgraduate Medical Institute, Lahore, Pakistan (Eth/ P609/FPGMI/2006).

Statistical analysis All statistical analyses were done with the SPSS statistical software package (version 15.0; SPSS, Chicago, USA). Statistical tests were all two-sided $(<0.05)$. Significances of differences between mean and median analytes of thiamine treatment and placebo groups were determined using Student's $t$ test and a Mann-Whitney $U$ test, respectively. Significances of differences from baseline and post-therapy and post-washout period mean and median analytes were determined using a paired samples $t$ test and a Wilcoxon signed-rank test, respectively. Correlation analysis was performed by calculating Spearman's rho statistic. Significance of difference of proportions was made by reference to Finney's $2 \times 2$ contingency tables.

Power calculation The primary endpoint was UAE. Group $\mathrm{CV}$ values and intervention effects of $30 \%$ were assumed, similar to previous studies [6-8]. For power $=0.8$ and $\alpha<$ 0.05 , patient group size was 17 . Patient groups of 20 were employed to allow for non-compliance to therapy.

\section{Results}

Patient characteristics at baseline The characteristics of type 2 diabetic patients with microalbuminuria recruited for this study at baseline are given in Table 1 . There was no significant difference in any clinical chemical variable at baseline. The median plasma thiamine concentration of diabetic patients was $7.5 \mathrm{nmol} / \mathrm{l}$, markedly lower than the normal range of normal healthy human individuals $(44.6-93.7 \mathrm{nmol} / \mathrm{l})$ [5]. The median urinary thiamine excretion was $1.38 \mu \mathrm{mol} / 24 \mathrm{~h}$. One patient had a plasma thiamine concentration above the normal range and a urinary thiamine output 30 -fold greater than the normal mean [5], consistent with prior thiamine supplementation, and was excluded from the data analysis. 
Table 1 Clinical data before and after 3 months of thiamine treatment and after a 2 month washout period

\begin{tabular}{|c|c|c|c|c|c|c|}
\hline \multirow[t]{2}{*}{ Variable } & \multicolumn{3}{|l|}{ Thiamine } & \multicolumn{3}{|l|}{ Placebo } \\
\hline & Baseline & Therapy & Washout & Baseline & Therapy & Washout \\
\hline $\begin{array}{l}\text { Plasma thiamine } \\
(\mathrm{nmol} / \mathrm{l})\end{array}$ & $10.6(0.8-84.5)$ & $98.2(2.6-294.5)^{\mathrm{c}, \mathrm{f}}$ & $10.9(3.6-22.7)^{\mathrm{i}}$ & $7.1(1.1-31.3)$ & $7.1(2.4-16.6)$ & $9.1(4.2-16.3)$ \\
\hline $\begin{array}{l}\text { Urinary thiamine } \\
(\mu \mathrm{mol} / 24 \mathrm{~h})\end{array}$ & $1.63(0.45-7.66)$ & $47.69(0.40-182.79)^{\mathrm{c}, \mathrm{f}}$ & $1.42(0.10-9.72)^{\mathrm{i}}$ & $1.53(0.67-4.46)$ & $1.71(0.15-9.73)$ & $1.16(0.00-5.44)$ \\
\hline $\begin{array}{l}\text { Thiamine clearance } \\
(\mathrm{ml} / \mathrm{min})\end{array}$ & $112(8-819)$ & $273(3-789)^{\mathrm{d}}$ & $92(18-237)^{\mathrm{h}}$ & $189(26-955)$ & $149(12-663)$ & $102(12-334)^{\mathrm{e}}$ \\
\hline $\mathrm{UAE}(\mathrm{mg} / 24 \mathrm{~h})$ & $43.7(33.0-120.9)$ & $30.1(12.0-38.2)^{\mathrm{b}, \mathrm{f}}$ & $20.9(7.0-35.0)^{\mathrm{f}, \mathrm{h}}$ & $50.9(32.9-121.7)$ & $35.5(6.4-82.0)$ & $30.0(3.5-80.4)^{\mathrm{f}}$ \\
\hline $\begin{array}{l}\text { Plasma glucose } \\
(\mathrm{mmol} / \mathrm{l})\end{array}$ & $10.1 \pm 3.2$ & $10.1 \pm 3.9$ & $8.0 \pm 3.5^{\mathrm{d}}$ & $9.5 \pm 3.1$ & $8.8 \pm 2.4$ & $8.0 \pm 3.1^{\mathrm{d}}$ \\
\hline $\mathrm{HbA}_{1 \mathrm{c}}(\%)$ & $9.2 \pm 1.3$ & $9.0 \pm 1.8$ & $7.8 \pm 1.6^{\mathrm{e}, \mathrm{h}}$ & $8.8 \pm 1.8$ & $8.5 \pm 1.7$ & $8.4 \pm 1.7$ \\
\hline $\begin{array}{l}\text { Total cholesterol } \\
(\mathrm{mmol} / \mathrm{l})\end{array}$ & $5.26 \pm 1.30$ & $4.87(2.59-9.69)$ & $4.61(3.29-7.20)$ & $4.79 \pm 1.06$ & $4.43 \pm 1.66$ & $5.57 \pm 1.97^{\mathrm{h}}$ \\
\hline $\begin{array}{l}\text { LDL-cholesterol } \\
(\mathrm{mmol} / \mathrm{l})\end{array}$ & $2.75 \pm 1.22$ & $2.56 \pm 1.71$ & $2.41 \pm 0.83^{\mathrm{a}}$ & $2.75 \pm 0.93$ & $2.38 \pm 1.19$ & $3.19 \pm 1.24^{\mathrm{g}}$ \\
\hline $\begin{array}{l}\text { HDL-cholesterol } \\
(\mathrm{mmol} / \mathrm{l})\end{array}$ & $1.29 \pm 0.35$ & $1.08 \pm 0.37^{\mathrm{d}}$ & $1.15 \pm 0.24$ & $1.24 \pm 0.27$ & $0.97 \pm 0.23^{\mathrm{f}}$ & $1.37 \pm 0.56^{\mathrm{h}}$ \\
\hline $\begin{array}{l}\text { Triacylglycerol } \\
(\mathrm{mmol} / \mathrm{l})\end{array}$ & $2.23(0.60-8.31)$ & $2.27(0.92-6.97)$ & $2.60(0.89-8.27)$ & $1.51(0.82-4.24)$ & $1.79(0.63-4.02)^{\mathrm{d}}$ & $1.97(1.08-4.46)^{\mathrm{d}}$ \\
\hline $\begin{array}{l}\text { Systolic BP } \\
(\mathrm{mmHg})\end{array}$ & $126 \pm 14$ & $133 \pm 20$ & $135 \pm 14^{\mathrm{d}}$ & $131 \pm 10$ & $130 \pm 17$ & $130 \pm 13$ \\
\hline $\begin{array}{l}\text { Diastolic BP } \\
(\mathrm{mmHg})\end{array}$ & $87 \pm 8$ & $85 \pm 9$ & $87 \pm 7$ & $84 \pm 6$ & $84 \pm 8$ & $83 \pm 7$ \\
\hline GFR (ml/min) & $85 \pm 19$ & $90 \pm 30$ & $77 \pm 20$ & $93 \pm 23$ & $97 \pm 18$ & $87 \pm 21$ \\
\hline sVCAM1 (ng/ml) & $588 \pm 267$ & $554 \pm 224$ & $481 \pm 192^{\mathrm{d}, \mathrm{g}}$ & $648 \pm 255$ & $608 \pm 178$ & $600 \pm 232$ \\
\hline vWF (IU/ml) & $0.60(0.07-5.18)$ & $0.48(0.01-2.95)$ & $0.51(0.071-1.97)$ & $0.48(0.01-4.35)$ & $0.48(0.01-4.41)$ & $0.59(0.01-1.61)$ \\
\hline
\end{tabular}

Data are means \pm SD or medians (minimum-maximum)

UAE was measured by immunoturbidimetry (Randox, Crumlin, UK) and GFR (serum creatinine with the Crockcroft-Gault formula) by the automated Jaffé method (Dimension Clinical Chemistry Analyzer; Dade Behring, Marburg, Germany). Fasting plasma glucose, total cholesterol, HDL-cholesterol and triacylglycerol were determined by the Dimension Clinical Chemistry Analyzer method, and $\mathrm{HbA}_{1 \mathrm{c}}$ by an agglutination inhibition rate assay (Randox). Serum LDL-cholesterol was deduced from the Friedewald equation. sVCAM-1 and vWF were measured by immunoassay kits (R\&D Systems, Abingdon, UK; Technoclone, Dorking, UK). Plasma and urinary thiamine was determined by pre-column derivatisation to thiochrome and HPLC with fluorimetric detection [5]

${ }^{\mathrm{a}} p<0.05,{ }^{\mathrm{b}} p<0.01,{ }^{\mathrm{c}} p<0.001$ compared with placebo; ${ }^{\mathrm{d}} p<0.05,{ }^{\mathrm{e}} p<0.01,{ }^{\mathrm{f}} p<0.001$ compared with baseline; ${ }^{\mathrm{g}} p<0.05,{ }^{\mathrm{h}} p<0.01,{ }^{\mathrm{i}} p<0.001$ compared with post-therapy

At baseline, there was a negative correlation of plasma thiamine concentration with thiamine clearance $(r=-0.52$; $p<0.01)$ and a positive correlation of urinary thiamine excretion and thiamine clearance $(r=0.52 ; p<0.01)$. There was no significant difference in the plasma thiamine concentration, urinary thiamine excretion and renal clearance of thiamine for patients with and without antihypertensive therapy. There were negative correlations of plasma thiamine concentration and urinary thiamine excretion with plasma von Willebrand factor (vWF) $(r=-0.41$ and -0.42 , respectively; $p<0.05)$. There was a significant negative linear regression of soluble vascular cell adhesion molecule-1 $(\mathrm{sVCAM}-1)$ on plasma thiamine $[\mathrm{sVCAM}-1(\mathrm{ng} / \mathrm{ml})=687-$ $(5.5 \times$ plasma thiamine $(\mathrm{nmol} / \mathrm{l})) ; p<0.05]$.

Effect of therapy with high-dose thiamine Thiamine treatment for 3 months increased the median plasma concentration of thiamine by tenfold and urinary excretion of thiamine by 29-fold. All liver function tests were within the normal range, indicating the expected absence of adverse effects of treatment with thiamine (Table 1).

The primary endpoint of this study was effect on UAE. After the treatment period, patients receiving thiamine therapy had decreased median UAE, with respect to patients receiving the placebo ( $30.1 \mathrm{vs} 35.5 \mathrm{mg} / 24 \mathrm{~h} ; p<0.01)$. UAE was decreased significantly with respect to baseline in the patients treated for 3 months with thiamine $(-17.7 \mathrm{mg} / 24 \mathrm{~h}$; $p<0.001)$ but not in patients treated with placebo. The decrease was maintained after the washout period when the decrease in urinary albumin in the patients treated with placebo also became significant (Table 1). The time course of changes in UAE in patients treated with thiamine and placebo showed a progressive decrease in UAE with time throughout the treatment period. Linear regression of UAE 


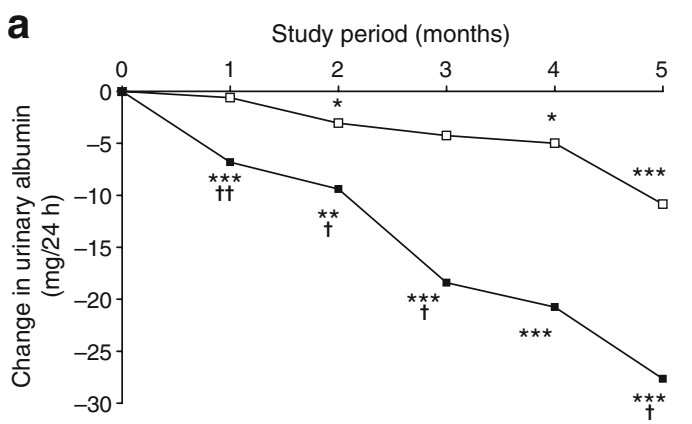

b

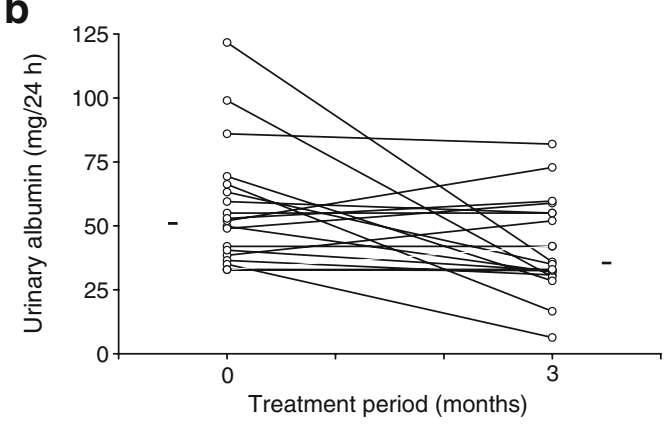

C

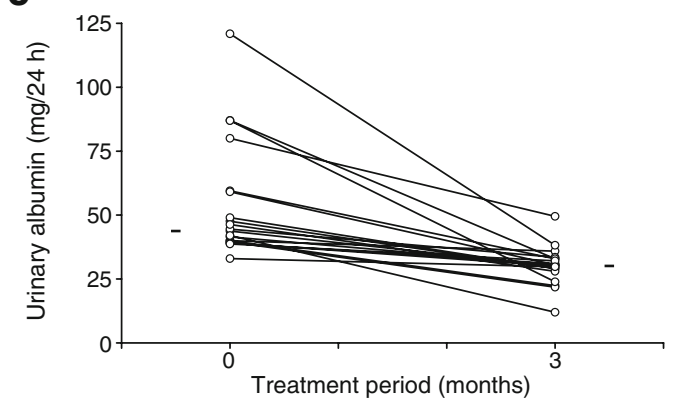

Fig. 1 Effect of high-dose thiamine on UAE. a Change from baseline of UAE in type 2 diabetic patients receiving thiamine (black squares) or placebo (white squares). Median values are shown: ${ }^{*} p<0.05,{ }^{* *} p<0.01$ and $* * * p<0.001$ compared with baseline; ${ }^{\dagger} p<0.05$ and ${ }^{\dagger \dagger} p<0.01$ compared with baseline change for the placebo. b Individual changes in UAE of patients on placebo; $p>0.05$. $\mathbf{c}$ Individual changes in UAE of patients on thiamine; $p<0.001$. Horizontal bars are median values

in relation to treatment time indicated that the rate of decrease in UAE was increased about fourfold in patients treated with thiamine with respect to placebo (5.62 vs $1.52 \mathrm{mg} / 24 \mathrm{~h}$ ) (Fig. 1).

There was no significant effect of thiamine supplementation on glycaemic control, dyslipidaemia, BP, GFR or markers of vascular dysfunction during the treatment period. After the washout period, fasting plasma glucose was decreased with respect to baseline levels in the thiamine treatment and placebo groups and $\mathrm{HbA}_{1 \mathrm{c}}$ was decreased with respect to baseline and 3 months after therapy in the thiamine treatment group. At this time, total cholesterol and LDL-cholesterol increased in patients treated with placebo, and LDL-cholesterol decreased in patients who had received thiamine therapy. HDL-cholesterol decreased significantly from baseline after the 3 month treatment period in patients treated with both thiamine $(16 \%)$ and placebo (22\%). HDL-cholesterol returned to baseline values in both groups after the washout period. Patients receiving placebo showed significant increases in triacylglycerol from baseline after the 3 month treatment and washout periods $(+18 \%$ and $+30 \%$, respectively). Patients receiving thiamine had a significant increase in systolic BP after the washout period. After the washout period, there was also a decrease from baseline concentration of SVCAM-1 in patients receiving thiamine with respect to placebo $(-117 \mathrm{ng} / \mathrm{ml}$; $p<0.05$ ) (Table 1).

\section{Discussion}

In this study we found that high-dose thiamine therapy $(3 \times 100 \mathrm{mg} /$ day $)$ decreased UAE in type 2 diabetic patients with microalbuminuria from baseline values after 1-3 months of therapy. After therapy for 3 months, regression of microalbuminuria to normal urinary albumin had occurred in $35 \%$ of the patients. Increasing the plasma concentration of thiamine in diabetes may reverse dysfunction of glomerular endothelial cells, podocytes and tubular epithelial cells thereby improving glomerular and tubular structure and function and low-grade vascular inflammation and decreasing UAE [9].

The baseline characteristics of the patients recruited for this study confirmed our previous findings that the plasma thiamine concentration is abnormally low in type 2 diabetic patients with microalbuminuria and with an inverse association of plasma thiamine with sVCAM-1 [5]. There was also a significant negative regression on plasma thiamine concentration and negative correlation of plasma vWF. Urinary excretion of thiamine $>0.20 \mu \mathrm{mol} / 24 \mathrm{~h}$ indicates adequate dietary thiamine sufficiency for adult human individuals [10]. On this basis, all diabetic patients in this study had an adequate dietary intake of thiamine.

Further studies are now required to confirm this encouraging pilot-scale outcome that high-dose thiamine reverses early-stage nephropathy in type 2 diabetes.

Acknowledgements We thank F. Ghafoor (clinical biochemist) for technical assistance and S. R. Naz, W. Ullah (senior medical officers), N. Akhtar (head diabetes nurse), Z. Anwaar and N. Sardar (staff nurses), I. Aziz (research assistant, sample cataloguing and dispatch), Diabetes Centre, Department of Medicine, Sheikh Zayed Hospital, Lahore, for assistance in patient recruitment. This study was funded by a project grant from the Pakistan Higher Education Commission. J. R. Larkin thanks the University of Warwick for a PhD studentship and N. Rabbani thanks the British Heart Foundation for an Intermediate Research Fellowship.

Duality of interest The authors declare that there is no duality of interest associated with this manuscript. 


\section{References}

1. Donnelly R, Emslie-Smith AM, Gardner ID, Morris AD (2000) $\mathrm{ABC}$ of arterial and venous disease-vascular complications of diabetes. BMJ 320:1062-1066

2. Thornalley PJ (2005) The potential role of thiamine (vitamin B1) in diabetic complications. Curr Diabetes Res 1:287-298

3. Babaei-Jadidi R, Karachalias N, Ahmed N, Battah S, Thornalley PJ (2003) Prevention of incipient diabetic nephropathy by high dose thiamine and benfotiamine. Diabetes 52:2110-2120

4. Babaei-Jadidi R, Karachalias N, Kupich C, Ahmed N, Thornalley PJ (2004) High dose thiamine therapy counters dyslipidaemia in streptozotocin-induced diabetic rats. Diabetologia 47:2235-2246

5. Thornalley PJ, Babaei-Jadidi R, Al Ali H et al (2007) High prevalence of low plasma thiamine concentration in diabetes linked to marker of vascular disease. Diabetologia 50:2164-2170
6. Sasso FC, Carbonara O, Persico M et al (2002) Irbesartan reduces the albumin excretion rate in microalbuminuric type 2 diabetic patients independently of hypertension: a randomized double-blind placebo-controlled crossover study. Diabetes Care 25:1909-1913

7. Andersen S, Brochner-Mortensen J, Parving HH (2003) Kidney function during and after withdrawal of long-term irbesartan treatment in patients with type 2 diabetes and microalbuminuria. Diabetes Care 26:3296-3302

8. Zandbergen AAM, Baggen MGA, Lamberts SWJ, Bootsma AH, de Zeeuw D, Ouwendijk RJT (2003) Effect of losartan on microalbuminuria in normotensive patients with type 2 diabetes mellitus: a randomized clinical trial. Ann Intern Med 139:90-96

9. Toyoda M, Najafian B, Kim Y, Caramori ML, Mauer M (2007) Podocyte detachment and reduced glomerular capillary endothelial fenestration in human type 1 diabetic nephropathy. Diabetes 56:2155-2160

10. Finglas PM (1993) Thiamin. Int J Vitam Nutr Res 63:270-274 